Their numbers varied greatly in the different reference was made to " congenital dacryocases and in different places in the lesions. In cystitis," when Dr. Knapp, Sr., made the stateand about many areas of necrosis none at all ment that these cases (dacryocystitis in infants) could be found. When present in a given area, never require treatment, - nature taking care they were usually few in number, but in Case I, of the difficulty in due time. I wonder if this in an area of degenerated hyperplastic intima, is the experience of members of this society. they were present in enormous numbers through- In my own it has been largely so, and yet out a portion of the area (see Fig. 3), and in only recently I was obliged to interfere in the Case II they were fairly numerous. In Cases case of a child ten months old, suffering from III and IV they were found only in small num- obstruction of the duct and blennorrhea of the bers and atypical forms of the micro-organism sac. I would remark in passing that this term, predominated. In Case V only atypical forms " congenital dacryocystitis," is unfortunate, for were found in small numbers. Some of these the sac is, I think, never inflamed at birth, this atypical forms were of the same length and inflammation being always secondary to the width as the typical treponema, but had less morbid process set up within the canal itself. numerous and less abrupt curves or windings In this particular case (and I select a clinical and sometimes presented a granular appear- case as the most convenient and concise way of ance; others were like the typical treponema presenting treatment for other similar cases), except that they were shorter. Inasmuch as the blennorrhea began within a week after birth, we found these atypical forms, not only along with the typical treponema in the other cases, but also in the liver of congenital syphilis among abundant typical forms, we consider them to be degenerated forms of the treponema. That they are not fragments of the tissue fibrils we have convinced ourselves after comparing them with such products in the same sections.

From the study of the microscopic appearances in these lesions, we consider the process to be one of primary necrosis of the media, and probably also of the intima, with the development of reparative connective tissue, followed by secondary degeneration and necrosis of this connective tissue. The finding of the treponemata in an area of primary necrosis in the media, as well as in association with the necrosis in the fibrous tissue, seems to justify the belief that the micro-organisms are the cause of the necrosis by their local action upon the tissue, and that they are to be regarded as the cause of the whole process, although the possibility that they are merely secondary invaders cannot be denied. Their numbers and distribution in the lesions would suggest that they rapidly multiply at a given point, produce necrosis and then degenerate and disappear.

Although the micro-organisms have the same morphology as the treponema pallidum, we are not thoroughly convinced that they are identical with that parasite.

A search through the literature revealed only two cases of syphilitic aortitis in which treponemata were found in the aortic wall. ${ }^{1}$

\title{
TREATMENT OF OBSTRUCTION OF THE LACHRYMAL DUCT.*
}

BY JOHN C. BERRY, M.D.

Ophthalmic and Aural Surgeon, Worcester City Hospital; former Surgeon Doshisha University Hospital, Kyoto, etc.

$$
\text { I. IN INFANTS. }
$$

DURING an animated discussion on dacryocystitis in the Ophthalmological Section of the British Medical Association at Toronto, in 1906,

1 Reuter: Zeitschr. für Hyg., 54, 1907, p. 49; Schmorl: Münch. med. Wochenschr., 1907, p. 188 .

${ }^{*}$ Read before the New England Ophthalmological Society, Boston. Jan. 12, 1909 .

and though mild disinfecting collyria were used, pressure made over the sac to force its contents on into the nose, and alkaline-antiseptic nasal sprays employed daily, yet the morbid process continued, and, indeed, grew worse, producing congestion and swelling of surrounding tissue. Fearing that a phlegmon would result if the case was allowed to pursue its course further, the child was put under an anesthetic, the canaliculus partially slit and a No. 3 olive-tipped probe passed on through the canal, a single passage being sufficient, as is usual, to completely relieve the difficulty. In these infantile cases the growth of micro-organisms is favored within the canal by the retained débris of fetal life therein, resulting in inflammation and softening of the lining membrane, thus making the passage of a small probe, especially one with a stiff shank, somewhat hazardous. I therefore hesitate to use a probe smaller than the No. 3, and an olivetipped probe with a small flexible neck that will easily bend and allow the tip to follow the canal is favored. It should be remembered that the course of the canal in babyhood is quite irregular and radically different from that in the adult, the direction being backward, at times, to an angle of nearly forty-five degrees.

These cases of dacryocystitis in infants are almost always from obstruction, - the abnormal proliferation of the epithelium of the canal obstructing its lumen, - especially at the lower end of the duct, which is very small at birth and further obstructed by pressure from the lower turbinate. Such pressure and occlusion produces stagnation in the canal itself, with resulting decomposition of its contents. Under such a condition it requires but a few days' exposure of the eyes to atmospheric conditions for the lachrymal secretion with micro-organisms from the eyelids and surface of the eyeball to be forced on into the sac and the whole gelatinous mass to become the seat of decomposition, inflammation and the appearance of the characteristic discharge of dacryocystitis at the inner canthus of the eye. According to the report of the London Ophthalmic Hospital for last January, there have been found in these discharges 
from infants, gonococci, staphylococci, pneumococei and Morax-Axenfeld bacilli.

In view of the fact that the obstruction at the nasal end of the canal is the chief etiological factor in these cases, it is possible that quick aspiration with a rubber bulb and nasal tip to fit the nostril would open the passage. I have not, however, known of this method being tried. Zentmeyer quotes Van Duyse as having seen " a lachrymal tumor rapidly relieved by the nurse applying mouth suction to the nose of the infant."

As further illustrating the nasal origin of this affection and the unusual treatment that may be required, I would refer to a very persistent case of infantile obstruction, with resulting dacryocystitis, which came under my care in September, 1906. The child was five years of age and had had profuse purulent discharge from both lachrymal sacs from a week after its birth. He had received faithful treatment at different times from two thoroughly competent men, oculists who evidently believe in the use of the large lachrymal probe, for as I examined the enlarged canaliculi I found them so dilated as to readily receive a No. 8 probe, which literally fell to the bottom of the canal. When inserting a probe for the first time it is my habit to throw a strong light into the nostril, well dilated, and see if I can discern the tip of the probe (frequently possible in adults), or, if not visible, then to seek to touch it with a nasal probe. In this case I could do neither. Forcing the lachrymal probe well downward and forward, I finally succeeded in bringing its tip within my field of vision, when I found it to be covered by a tough membrane, the lachrymal canal thus being impervious, while in the opposite nostril the anterior end of the lower turbinate was firmly adherent to the wall, thus concealing everything in this nostril and rendering necessary the removal of the anterior portion of the lower turbinate. This was done at once, and brought plainly into view a similar membrane covering the lower end of this canal. With a pair of Hartman's cutting-edge forceps I seized and took away that portion of this membrane covering the end of the lachrymal probe, and then pushed the probe on into the nasal cavity. In the case of the left duct this was at once successful, little further treatment being required save the daily cleansing of the duct and nasal cavity and the injection into the canal of a two per cent solution of ichthargan.

With the opposite nostril, however, more trouble was experienced, the membranous obstruction reforming at the nasal end of the canal even after being twice removed. To insert a style that should reach quite through to the floor of the nostril, thus including the lower portion of the canal, seemed inadvisable because of the inflammation it would occasion by pressure on the floor itself, and I therefore resorted to the use of a seton. With the child under ether, I passed a small, flexible probe, olive tipped at one end, and with an eyelet armed with several threads of sterile floss silk at the other, on through the canal. With a pair of alligator nasal forceps and good illumination it was easy to seize this probe as it appeared in the nostril, bend it sharply upon itself and thus work it downward gradually through the canal and out through the nostril. In the case of an adult with the nostril larger and the canal directed more anteriorly, this would doubtless be easier. It was not difficult, however, in this five-year-old boy. The projecting ends of the silk were brought together and tied, and the whole held safely along the side of the nose by surgeon's plaster. The seton was cleansed with boric acid solution, impregnated with a $2 \%$ solution of ichthargan, and moved every day. At the end of a week it was judged that the opening had become permanently patulous, and the seton was, therefore, removed. The discharge soon ceased under a simple boric acid cleansing and application of ichthargan, and the child has remained well ever since. This case is especially suggestive as emphasizing both the nasal origin of these obstructions, which is usual, and the continued nasal seat of the difficulty, there being no stricture in the duct itself. I have, therefore, referred to it somewhat at length as illustrating (1) the necessity of intranasal work in some of these cases of obstructive blennorrhea and (2) the possibilities of the seton when a rebellious tendency to reclosure of the canal is experienced.

\section{IN ADULTS.}

Of the absolute necessity for operative relief in cases of dacryocystitis in adults there can be no possible question. The argument of the patient that the discharge has existed for years without injury to the eye should not be allowed to weigh for a moment. True, the conjunctiva is very tolerant of the presence of this discharge, loaded, though it is, with various bacilli, but should there occur any injury to the cornea, either traumatic or idiopathic, then grave danger immediately arises. A knowledge of this fact on the part of the patient usually removes all objection to operative interference for relief.

Whether the troubles arise from obstruction or from stricture, we should seek to relieve it by gradual dilation rather than by cutting the stricture and rapid dilation. We should not hesitate, therefore, to slit up the lower canaliculus and pass a probe, usually a No. 4 or No. 5 , olive tipped, following this, in three days, with one of larger size, and so on, every third day, until a No. 8 , or possibly 10 , can be easily received, always allowing the probe to remain in position about fifteen minutes. To allay the pain incident to this pressure, the probe may be anointed with a sterile ointment containing cocaine, in the hope that a little of this may reach the entire canal. Of course an aqueous solution of cocaine with a slight amount of adrenalin chloride is first injected into the sac and canal as far as the obstruction will permit it to pass before the probe is introduced. In all manipulations within the sac or the canal we should guard against injury to the membrane, preferring a gradual dilation to a rapid and violent one, lest the cicatrices resulting from injury contract the lining membrane of the canal and further restrict its lumen. Even in a case of stricture I defer making use of the knife for its division until I have made faithful attempt for cure by stretching and gradual dilation. As the stricture is caused by chronic inflammation, with consequent thickening of the lining membrane of the canal, of the periosteum, or, it may be, of the bony substance beneath, it is rational to encourage its absorption by pressure, and to this end we should allow a probe of adequate size to remain in situ for 
a number of minutes, fifteen or twenty, as above stated. Beyond a No. 8 probe I seldom go, unless $I$ have to divide the stricture, when I follow the division with a conical probe and enlarge the canal at once, to size No. 14 if possible, later using the smaller probes of 8 or 10 . In doing this little operation I use an angular knife, probe tipped, and cut the stricture in three directions that, as emphasized by Harrower in a paper before our state society, the contracting cicatrices may draw apart the walls of the stricture rather than further narrow it, as in the case of a cicatrix from a single cut. I am aware that many eminent authorities favor the use of the larger probe in the regular work of stretching, using great force, if necessary, to introduce it, regardless of resulting traumatism within the canal. I have employed both methods and favor the more conservative course. I would not at this time raise lengthy discussion on a point on which able minds have found it so difficult to agree. I simply give the results of personal observation when I say that a medium dilation is sufficient for the passage of tears; avoids traumatism; exposes less the membrane within the canal, and the eye, to contamination by nasal secretion; guards against regurgitation of the contents of the tear duct when blowing the nose; preserves the propulsive action of the lachrymal sac, so necessary to the passage of its contents on into and through the duct; and, apparently, is not followed by a return of the stricture in any larger proportion of cases than when the maximum dilatation is carried out.

A point much emphasized by the supporters of forced and maximal dilation is the absorption of hypertropied tissue by pressure - mucous membrane, periosteum and even bony tissue itsclf. My recent practice in such cases of rebellious hypertrophy and obstruction is to secure this absorption by the wearing of a leaden style, after the suggestion of Moulton before the Section of Ophthalmology of the American Medical Association held last June in Chicago. A style slightly smaller than the last probe introduced is used, thus enabling the patient to wear it with comfort and still have slight pressure exerted upon the walls of the canal between the treatments by probes. Three sizes of leaden wire can be obtained of most any

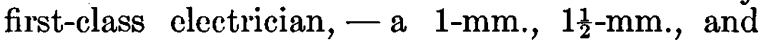
a 2 -mm. size. Here is a smaller one recently removed from a patient. This is a $1-\mathrm{mm}$. size. A No. 8 probe was passed with considerable difficulty before using this. The style was worn three weeks without the slightest inconvenience, and, when removed, this same No. 8 probe, previously passed with difficulty, was passed with the greatest ease. This is only a fresh illustration of a fact, long ago established, that continuous pressure will produce absorption of tissue, while interrupted pressure produces hypertrophy.

Before applying this leaden style the ordinary probe is inserted, then lifted to the level of the nasal orifice of the canal, and then, on withdraw- ing it, the leaden style is molded to take the curve of the probe, cutting it one fourth of an inch shorter than the exact length of the canal, as measured on the probe just withdrawn. The style is then bent sharply upon itself, giving the shoulder you here see, and which, when in place, lies completely concealed in the canaliculus. Its withdrawal is easily effected by hooking under this shoulder a Steven's small strabismus hook, while it can easily be inserted with a Prince's advancement forceps or an ordinary pair of epilating forceps. Of course a fixation forcep or any instrument with teeth would roughen the surface of the leaden style and cause it to irritate the membrane of the canal. As I have already intimated, the style will make increased room for itself, and, following this, a larger probe and style can be employed.

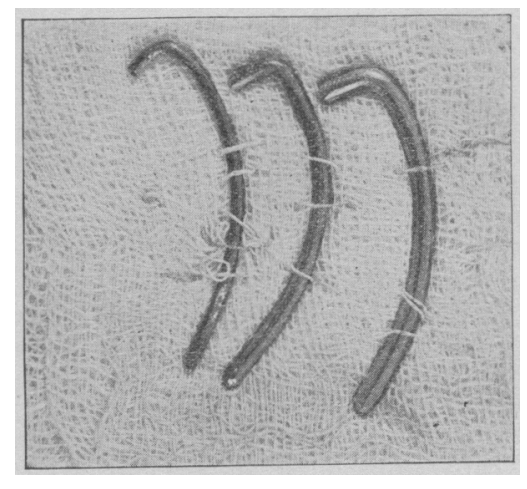

Leaden stylets, $1,1.5$ and $2 \mathrm{~mm}$. in diameter.

The experience with drainage tubes in these intractable cases of stricture has not been satisfactory, and the use of the leaden style, as proposed by Moulton, should, I think, have an extended trial. Even in those rebellious cases that resist ordinary treatment and seem to demand the extirpation of the sac, it would seem prudent to give fair trial to the leaden style, or to the seton, before resorting to more heroic measures. It is our imperative duty to care for our patient in the best way, preserving, if possible, the normal passage for the tears. Koster, as reported in the Annals of Ophthalmology for April, 1908, emphasizes this, reporting the cure of several intractable cases of strictural dacryocystitis treated by the seton. The use of this remedy two years ago, in my own case of obstruction, was certainly most satisfactory. Whether in hypertrophic stricture we could secure absorption by pressure as rapidly from the seton as from the leaden style I am in doubt. Of course one advantage of the seton is that remedial measures can be applied on the silk, as solution of silver nitrate, ichthargan, protargol, argyrol, etc., and thus be brought into touch with the affected membrane as the position of the seton is changed from day to day.

$$
\text { PHLEGMON. }
$$

When, in case of lachrymal obstruction, infection of the sac wall occurs, then an ab- 
scess is inevitable, unless it can be treated very early. In such cases, when seen early, my invariable rule is to slit up the canaliculus at once and enter the sac, if possible (we know there is an obstruction that will require this and the use of the probe later), then wash out the sac and introduce a $25 \%$ solution of freshly prepared argyrol. For this purpose, and, indeed, for all work on the lachrymal canal, I have never found any syringe quite equal to that of Risley's, made by Ferguson, of Philadelphia. Thus treated and followed up by the application of hot stupes of lead and laudanum, renewed every three hours and covered with protective rubber tissue or oiled silk, and retained by a bandage, the inflammation and swelling rapidly subside, making it usually safe to employ a probe at the end of five or six days.

In case the surrounding tissue beyond the sac has become infected to any considerable extent, an abscess will follow. This requires rational treatment and a more prolonged delay before the swelling can sufficiently subside to make safe the use of the probe.

Summarizing this brief review:

\section{IN INFANTS.}

1. Ordinary cases of dacryocystis in infants require no treatment other than nasal cleanliness, boracic acid collyria and slight pressure over the sac.

2. More persistent cases may require nasal aspiration or possibly the passage of the lachrymal probe.

3. Obstruction is sometimes due to membranous closure of the nasal end of the canal.

4. Intranasal inspection is always prudent and at times necessary when probing the duct.

5 . The seton is a remedial measure of great promise in certain rebellious cases.

\section{IN ADULTS.}

1. The ideal treatment is by gradual dilation, avoiding violence to the membrane of the canal.

2 . If this proves unsuccessful, then division of the stricture with rapid and full dilation is necessary.

3. Dilation beyond $2 \mathrm{~mm}$. (Bowman's Probe No. 8) is seldom necessary. Forced dilation to $4 \mathrm{~mm}$. (Theobold No. 16) exposes the eye to nasal contamination and lessens the propulsive power of the sac.

4. The leaden style can be employed to advantage between treatments, reducing the frequency of probing and favoring absorption of hypertrophied issue by continuous pressure.

5 . Acute cases of inflammation of the sac can be aborted if seen early, the treatment being to wash out the sac, inject argyrol and follow with hot stupes of lead and laudanum, general antiphlogistic treatment being observed.

6. Following phlegmon, the passage of the probe should be delayed until swelling and induration subside.

\section{ghałgarhusettg General Wogpital.}

CLINICAL MEETING OF THE MEDICAL BOARD, HELD IN THE TREADWELL LIBRARY. MARCH 26, 1909.

GANGRENE OF THE LUNG.

BY HERMAN F. VICKERY, M.D.

Av Italian laborer, A. d'O., forty-three years old, was admitted to my wards on Feb. 1, 1909. His family history, previous health and habits were unimportant. For over a year he had had cough with whitish expectoration. Six weeks before entrance he felt too weak to continue work; since then cough and expectoration had slowly increased. Twenty-four days before entrance he had fever for one day only, after that he had coughed up about one half a cupful of blood, and the sputum became bloody, extremely foul and copious, from 1 to 3 cupfuls a day.

Physical examination showed a well-developed and well-nourished man, with moist and coarse dry râles heard throughout the chest, but especially on the right side. There seemed to be slight dullness and increased voice sounds above and below the right clavicle, and in the same region the râles seemed coarser and more numerous than elsewhere.

On Feb. 15 an x-ray picture disclosed some opacity beneath the right clavicle and there was now an approach to amphoric breathing in the same region. The sputum amounted to $1 \frac{1}{2}$ cupfuls or more a day and repeated examinations disclosed no tubercle bacilli nor elastic fibers, nor any distinctive bacteria.

On the 14th of February the temperature, which had remained in the neighborhood of $99^{\circ}$, shot up to $104^{\circ}$ the right apex filled with high-pitched bubbling râles and shortly after the sputum became bloody. During the next nine days there were several such attacks, and the patient began distinctly to lose ground.

Treatment had consisted of constant exposure to fresh air, forced feeding, myrtol, oleoresin of cubebs, terebene, guaiacol and inhalations of compound tincture of benzoin. Morphine was occasionally necessary to control the cough.

It was felt that a gangrenous process was located in the right apex, but anatomical considerations deterred us from inserting an aspirator in this region. As the patient was evidently going from bad to worse we felt that surgical interference afforded him the only chance for recovery, and on Feb. $19 \mathrm{Dr}$. Scudder saw him in consultation and accepted him for subsequent operation. Dr. C. L. Scudder said that the gangrenous area was drained, the patient making a good recovery from the operation. He will report the case more fully later.

\section{ABSCESS AND GANGRENE OF THE LUNGS.}

$$
\text { BY F. T. LORD, M.D. }
$$

Some time ago I collected the $\operatorname{cases}^{1}$ of abscess and gangrene of the lungs which had come to autopsy or operation at the Massachusetts General Hospital. A brief consideration of the study of this series may be of interest in connection with the case reported by Drs. Vickery and Scudder.

Surgery is more likely to be successful when the pulmonary loss of substance is single. The history of the case, the physical signs and the use of the $\mathrm{x}$-ray may be expected to afford valuable evidence concerning the number of the lesions, but the chances are rather in favor 1.Reported in International Clinics, vol. ii, sixteenth series. 